\title{
KRIDANTA ANALYSIS FOR SANSKRIT
}

\author{
N. Murali ${ }^{1}$, Dr. R.J. Ramasreee ${ }^{2}$ and Dr. K.V.R.K. Acharyulu ${ }^{3}$ \\ ${ }^{1}$ Department of Computer Science, S.V. Vedic University, Tirupati \\ ${ }^{2}$ Department of Computer Science, R.S. Vidyapeetha, Tirupati \\ ${ }^{3}$ Professor of Vyakarana (Retd.), R.S. Vidyapeetha, Tirupati
}

\begin{abstract}
Kridantas play a vital role in understanding Sanskrit language. Kridantas includes nouns, adjectives and indeclinable words called avyayas. Kridantas are formed with root and certain suffixes called Krits. Some times Kridantas may occur with certain prefixes. Many morphological analyzers are lacking the complete analysis of Kridantas. This paper describes a novel approach to deal completely with Kridantas.
\end{abstract}

\section{KEYWORDS}

Avyaya, Kridanta, Morphological Analyzer, Natural Language Processing, upapada, upasarga

\section{INTRODUCTION}

Word is considered as the most basic unit of the linguistic structure. Word is a sequence of characters delimited by space. Word consists of a complex set of more primitive parts. The study of morphology is concerned with the construction of words from more basic meaningful units called morphemes. The process of analyzing the given word to extract the information encoded in the word is called as morphological analysis. Morphological analysis deals with the segregation of word into morphemes. By identifying the morphemes of a given word, the form (syntax) and meaning (semantics) of the word can be understood. Morphological Analyzer (MA) is a tool which analyzes the given word into its root, affixes and feature values of the grammar like number, gender, person etc.

\section{A. MAs for Sanskrit}

A thorough survey has been conducted on existing MAs and the following four MAs for Sanskrit language have been selected for the present study.

1. The Sanskrit Reader Companion developed at INRIA

2. MA developed at $\mathrm{HU}$

3. MA developed at JNU

4. MA developed at $\mathrm{UoH}$

The stories of Balakanda from Ramayana which were published in the children's fortnightly magazine called Chandamama has been collected and used as a test data to test the above mentioned MAs. The test data contains a total number of 28,713 words, out of which 8,981 words are unique words. The rich linguistic features of Sanskrit like Sandhi, Samasa, kridantas, taddithas etc., have great impact on the performance of the above mentioned MAs. Out of these, kridantas is one of the very interesting features of Sanskrit which includes nouns, adjectives and indeclinable words / avyayas. 


\section{KRIDANTAS}

In Sanskrit, the words can be broadly classified into 2 categories viz. Declinable and Indeclinable [2]. The following figure illustrates this [16].

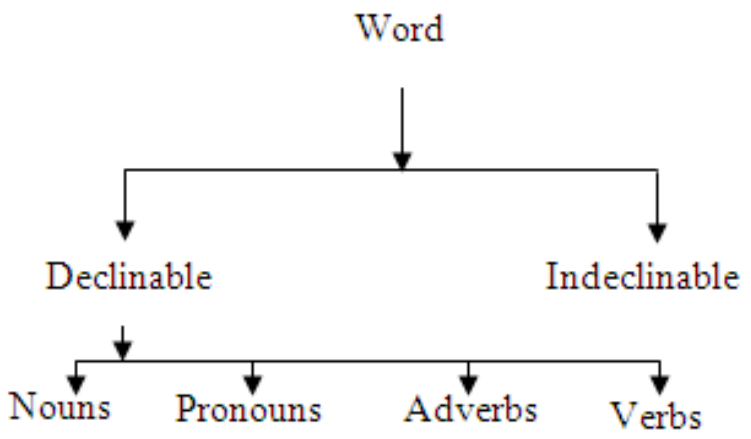

Figure 1. Classification of Words

Declinable or inflectional words are those words whose base form can be changed or inflected. For example, the base form or pratipadikam "Rama" can be inflected in 8 vibhaktis i.e., cases and three vacanas i.e., numbers. These declinable/inflectional words can again be categorized as Nouns, Pronouns, Adverbs and Verbs. In Sanskrit, indeclinable words are called avyayas.

Nouns and adjectives can be derived from verbs and this process is called nominalization. The term krit represents a process of nominalization in Sanskrit in which certain affixes can be coalesced with roots to generate nouns or adjectives or indeclinable words i.e., avyayas. The words which have been so generated are called as kridantas. These affixes are called krit suffixes. All these suffixes were described in the text siddhantakaumudi under a separate chapter called Kridanta prakaranam. Krit affixes are used to indicate karakas. The activity element possessed by the root lies inactive in the verbal derivative nouns and adjectives. In case of avyayas the root acts as an infinitive and directly related to the main verb in the sentence. All krit suffixes may be used in the senses of different karakas. Panini has mentioned that, if no other sense is assigned to a krit affix, then it should be understood that karta or agent of the verbal activity [1]. Certain upapadas and upasrgas may change the meaning of a kridanta completely. The following examples illustrate kridantas and shows how a root can be inflected in different ways with upapada or upasarga combinations.

Example 1: रामः (Ramah) - means who makes every one happy and is derived from

Verbal Root रम् (ram) and

Krit Suffix घ्, (Ghay)

Example 2: विरामः (Viramah) - means recession / rest / relaxing and is derived from

Prefix वि $(v i)$

Verbal Root रम् (ram) and

Krit Suffix घन् (Ghay)

Example 3: आरामः (Aramah) - means abode and is derived from

Prefix आ (A) 
$\begin{array}{lr}\text { Verbal Root } & \text { रम (ram) and } \\ \text { Krit Suffix } & \text { घन् (Ghay) }\end{array}$

Kridantas may be nouns, adjectives and avyayas. The Kridantas nouns and adjectives fall in the category of declinable words and avyaya falls in the category of indeclinable words. The nouns and adjectives are treated similarly. Hence the process of identification of Kridantas is divided into two stages

\author{
Identification of Avyaya and \\ Identification of Kridantas
}

Avyaya, types of avyayas, the process of recognizing avyayas and other kridantas have been presented in the following sections.

\title{
3. Avyayas
}

The following sloka gives a beautiful and simple definition for avyayas

$$
\begin{aligned}
& \text { सहशं त्रिषु लिए्गेषु सर्वासु च विभक्तिषु । } \\
& \text { वचनेषु च सर्वेषु यन्न व्येति तदव्ययम् ।। } \\
& \text { sadrusam trishu lingeshu srvasu ca vibhaktishu. } \\
& \text { vacaneshu ca sarveshu yanna vyeti tadavyayam. }
\end{aligned}
$$

The sloka connotes that the words which cannot be changed or inflected or which remain immutable in all genders, numbers and cases; are called avyayas [2], [6]. But, there is an exception to some nouns (Nominative or Locative), which have only one declension e.g. adau, samvat, svar, svaha, svadha etc. Figure 2 illustrates various types of avyaya [16]. Some grammarians have classified avyayas into Substantive and Non-substantive. The examples for Substantive avyayas are asti, adau, samvat, svar etc. Non-substantive avyayas can be further divided into two types i.e., Conditional and Relational. cet, yat-tat, yatha-tatha, yadi-tarhi, yatratatra, yavat-tavat etc. are the examples of Conditional avyayas, while atha, api, iti, iva, eva, evam, kila, khalu, ca, tu, manye, vat, va etc. for relational avyayas. For the present study, avyayas have been classified into two types i.e., Primitive/ Paniniyan avyayas and Secondary / Lakshanika avyayas.

\subsection{Primitive/ Paniniyan avyayas}

These types of avyayas are directly named by the Sanskrit grammarian Panini as nipatas. These avyayas can be used as prepositions, interjections, particles and conjunctions and can be called as nipatas i.e., exceptional or irregular forms. All nipatas are indeclinable. All upasargas / prefixes can also be treated as nipatas and are indeclinable.

\subsection{Secondary / Lakshanika avyayas}

These are used as adverbs or adjectives. Kridanta avyayas, taddhitanta avyayas and adverbial, determinative (of type pradi, gati) and attributive types of compounds fall into this category. 
International Journal on Natural Language Computing (IJNLC) Vol. 3, No.3, June 2014

\subsubsection{Kritpratyayanta words as avyayas}

Kritpratyayas are used to form nouns and avyayas from roots [2], [6]. These avyayas denote some action, which have a relation to the main verb in the sentence.

\subsubsection{Taddhitapratyayanta words as avyayas}

Ttaddhitapratyayas are those suffixes that are used to form nouns or avyayas from other nouns.

\subsubsection{Compounds as avyayas}

Some compounds such as Adverbial / avyayibhava, Determinative / tatpurusha (of type pradi and gati) and Attributive / bahuvrihi also act as avyayas.

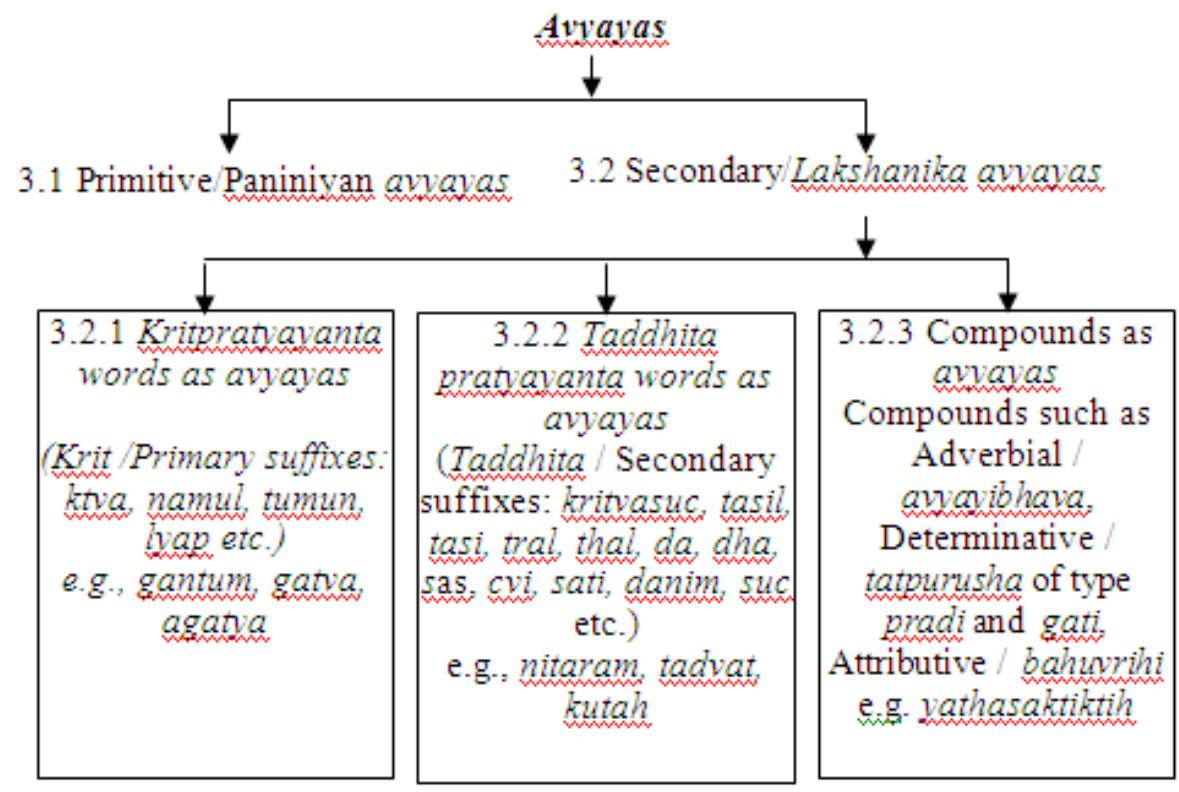

Figure 2. Classification of avyayas

\section{Avyaya Analyzer}

Avyaya Analyzer deals fully with the primitive type avyayas i.e., 3.1, and Kridantas i.e., 3.2.1 and partly with Taddhitas i.e., 3.2.2. Dealing with avyayas of primitive type is very simple because it has already been defined as avyayas by Panini in nipatas. Some of the examples of primitive type avyayas are asti, adau, atha, api, iti, iva, eva, evam, katham, kila, khalu, ca, tu, manye, vat, va etc. Here a simple lookup is enough to identify the avyaya of this category. The avyayas of type 3.2.2 had been encoded with information and play a vital role in understanding the sentence. Consider the following examples:

$$
\begin{aligned}
& \text { Gantum means "to go" } \\
& \text { Agantum means "to come" } \\
& \text { Gatva means "having gone" } \\
& \text { Agatya means "having come" }
\end{aligned}
$$

The above examples were derived from a single root gam, prefix "a" and krit suffixes tumun, ktva and lyap. Each word indicates a different meaning because of the suffixes. All these avyayas 
indicate incomplete action of their constituent verbal roots and have a relation to the main verb in the sentence and act as an adverb. The following simple sentences illustrates the role of avyayas Sentence: aham gantum icchami.

POS: Noun avyaya verb

Meaning: I wish to go.

Sentence: aham agantum icchami.

POS: Noun avyaya verb

Meaning: I wish to come.

Sentence: bhavan tatra gatva vadatu

POS: Noun avyaya avyaya verb

Meaning: having gone there, you tell.

Sentence: atra agatya upavisatu

POS: avyaya avyaya verb

Meaning: having come, sit here.

In all the examples given above, avyayas act as adverbs. Both the avyaya and main verb are mutually dependent on each other and it is impossible to understand the meaning of the sentence without gaining a proper understanding of the avyaya.

Hence the structure of avyaya may be as follows:

$$
\text { Avyaya } \rightarrow\left(\text { Upapada }^{*}\right)\left(\text { Upasarga* }^{*}\right) \text { root suffix }
$$

Upapada and upasarga are optional. The Avyaya Analyzer will give the derivational morphological information of an avyaya i.e., the upapada, upasarga, root and suffix information.

\subsection{Representation of linguistic information}

Avyaya Analyzer relates the root and linguistic features to the surface form through a set of transformations. 2000 verbal roots, 120 krit suffixes (out of which four suffixes are related to avyayas), 22 upasargas and nearly 500 upapadas were collected. With the combination of all these upapadas, upasargas, roots and suffixes, we can generate a large number of avyayas. The upapada, upasarga, root and suffixes acquire different forms due to euphonic transformations between them. Hence, each and every possible phonetic change of roots, suffixes, upasargas, and upapadas were written manually along with the necessary linguistic features. Morphological dictionaries for upapadas, upasargas, roots and suffixes were created. An example of the possible phonetic changes in upapada, upasarga, root and suffix are given below. For computational purpose, the data is presented in WX transliteration scheme. Converters are available to convert the text from WX transliteration to Devanagari Unicode UTF-8 and viceversa.

Upapada:

sva/sv,sva

IRax/IRan/IRac/IRaj/IRal/IRaw,IRaw

iram/iraM,ira

jala/janaM/janam/jala/jana/jal/jana, jana

Upasrga:

nir/niH/nil,nir

vi/vy/v,vi

anu/Anu/anv/Anv/onu/onv, anu

apa/ap/opa/op/Apa/Ap,apa 
Root:

$\mathrm{raMram} / \mathrm{riraM} / \mathrm{ram} / \mathrm{raM} / \mathrm{rAm} / \mathrm{ran} / \mathrm{rem} / \mathrm{raw} / \mathrm{ra}, \mathrm{ram} \_1 \_\mathrm{A} \_853$

iR/IR/eR/ER,iR_4_pa_1127

iR/IR/eR/ER,iR_6_pa_1351

krit suffix:

anIya/aNIya/nIya/NIya,anIyar

Davaw/iwavaw/Iwavaw/navaw/Navaw/tavaw/wavaw/ Xavaw,kwavawu

Contents of upapada, upasarga and krit suffix are separated with comma. The second field indicates the surface form of upapada / upasarga and the first field indicates all possible phonetic changes of the second field in context of various euphonic transformations. The second field in the Root gives the information about the root, class, type, and root number as given in siddhantakaumudi.

\subsection{Identification of avyayas}

AA checks whether the word is avyaya or not by noticing whether the word is nipata or by comprehending its suffix. If the word is nipata, then it will be treated as avyaya. If the word ends with any one of the krit suffixes related to avyaya, then it return suffix info and strips the suffix and checks for upapada [11]. As upapada and upasarga are optional, they may or may not be present in the given word. Hence, if upapada is present, then returns the upapada info and strips it. Then the presence of upasarga will be tested. If upasarga is found, then returns upasarga info and strips it. Then checks whether the remaining part of the word is the root or not. If it is, then return the root info; or else control will be transferred to the next stage. The following figure illustrates the process of analyzing the avyaya. 


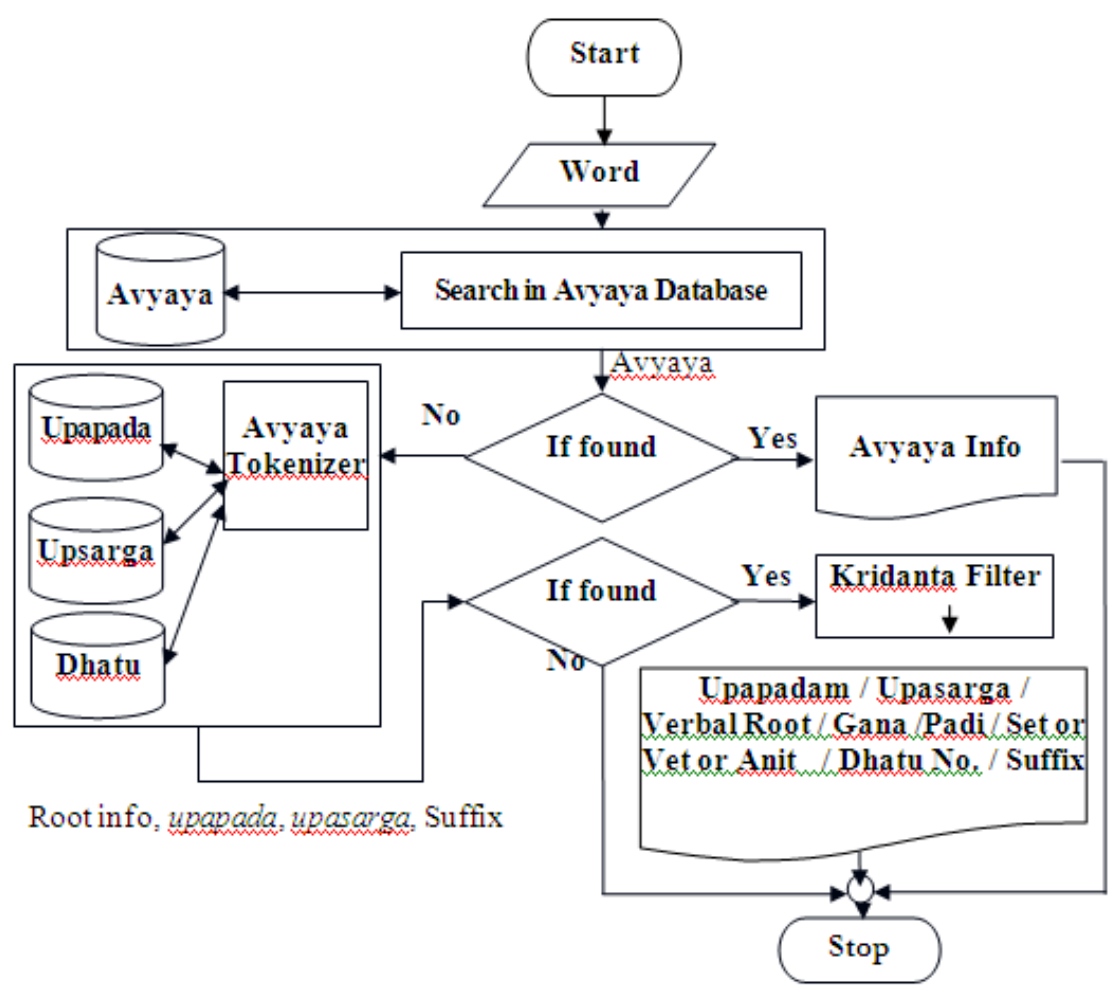

Figure 3. Avyaya Analyzer

The sample output of the Avyaya Analyzer in WX transliteration scheme is presented below. The output is rewritten for convenience.

Input Word: BaFjayiwum (bhanjayitum)

Output:

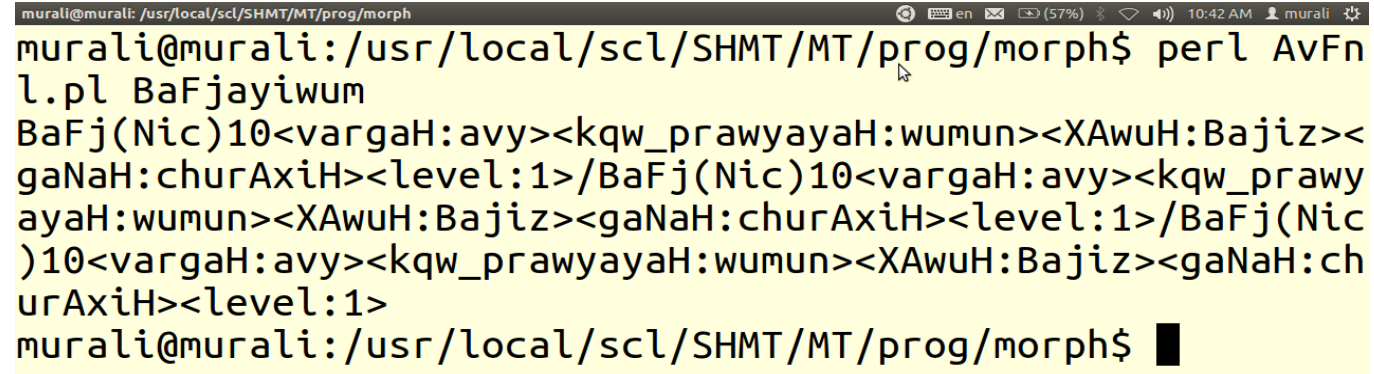

Figure 4. Output of the Avyaya Analyzer

Input Word: BojayiwvA / (bhojayitva)

Output: 

murali@murali:/usr/local/scl/SHMT/MT/prog/morph\$ perl AvFn l.pl BojayiwvA

Buj7<vargaH: avy $><k q w \_p r a w y a y a H: k w v A><X A w u H: B u j a z(N i C)><g a N$ aH: ruXAxiH $><$ level:1>/Buj7<vargaH:avy $><$ kqw_prawyayaH:kwvA $><$ XAwuH:Bujaz(NiC) $><$ gaNaH: $r u X A x i H><$ level: $1>/$ Buj7<vargaH:avy $>$ $<k q w \_$prawyayaH:kwvA $><X A w u H: B u j a z(N i c)><g a N a H: r u X A x i H><l e v e$ $l: 1>$ murali@murali:/usr/local/scl/SHMT/MT/prog/morph\$

Figure 5. Output of the Avyaya Analyzer

Input Word: lakRyIkqwya / lakshyikritya

Output:

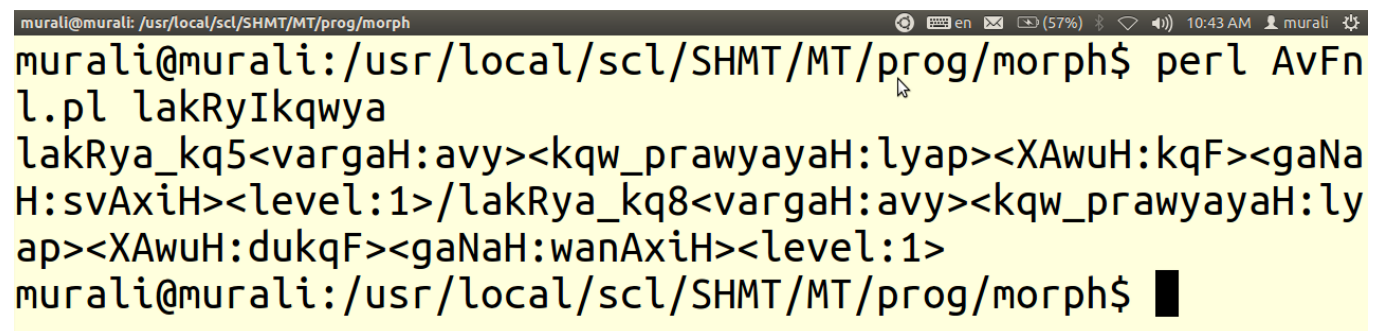

Figure 6. Output of the Avyaya Analyzer

\section{KRIDANTA RECOGNITION}

Kridantas have been discussed briefly in Section 2. Kridantas may be nouns or adjectives or ayayas. Some times a kridanta can be treated as a verb when there is no main verb in the sentence. The following example illustrates this.

Example: nirgawaH

The word mentioned above is in nominative case. It is derived from an upasrga-nir, root -gam, $k r i t$ suffix $-k w a$ and a sup suffix. This word functions as a verb. A word consisting of krit suffix like kta, ktavatu denotes the action done by the agent. In Sanskrit, adjectives will also take suffixes and can be inflected in all cases and in all numbers just like nouns. Hence, adjectives and nouns are treated in a similar way in the process of analyzing them. As it is pointed earlier, krit suffixes are used in the process of generating nouns, adjectives and avyayas. Avyayas cannot be inflected, but nouns and adjectives can be inflected by adding 21 sup pratyayas (suffixes) to the base form or pratipadika of a kridanta. The surface form of a kridanta will contain a root, krit pratyaya and a sup pratyaya. Upapada and Upasarga are optional.

$$
\text { kridanta } \rightarrow(\text { upapada*) (upasarga*) Root KritPratyaya SupPratyaya }
$$

A kridanta may contain any number of upapadas and upasargas. Sometimes a kridanta may contain another kridanta as upapada. Hence to analyze a kridanta properly, it is essential to know its base form or pratipadika, case, gender and number. The information regarding the case, gender and number will be useful in the process of identification of phrases and understanding the semantic role of each word. The process of identification of kridantas is described below. 


\subsection{Identification of Pratipadikam}

The process of reducing the inflected form to its stem is known as stemming [8]. For example, in English, a stemmer should identify the word "cats" to the stem "cat". In 1968, Julie Beth Lovins published the stemmer [3] and later Stemmer was written by Martin Porter [4] for English. The stemming process for Sanskrit was described here. In Sanskrit the stem is called as "pratipadikam". In Sanskrit the process of identifying the base form of an adjective is similar to that of a noun. The base form of a noun can be identified by removing the sup pratyaya from the surface form. By drawing some clues from the Porter's stemming algorithm, a stemmer for Sanskrit has been developed to retrieve the base form along with the linguistic features like gender, case and number.

Generally a student who wants to learn Sanskrit will start with learning the basic noun forms like rAma, ramA etc. The remaining nouns will be similar to these basic noun patterns. A Sanskrit basic text called Roopchandrika [5] consists of 206 noun types or patterns. It gives basic information regarding gender, word forms in eight cases and three numbers (Singular, Dual and Plural numbers) for each word and totally each stem has 24 inflections. In Sanskrit, all nouns and adjectives follow any one of the pattern of intonation. Based on high frequency of usage, only 23 stems have been selected among the 206 stems and are given in the table 1 . The information regarding the inflections, gender, suffix, case, and number was stored in a table which will be used as Paradigms [9], [10]. The Suffix information was stored separately in a manner which will be useful in stripping the suffixes.

Table 1: Words used in designing paradigm table

\begin{tabular}{|c|l|l|}
\hline Sl. No. & Word & Gender \\
\hline 1. & ramA & F \\
\hline 2. & mawi & F \\
\hline 3. & naxI & F \\
\hline 4. & lakRmI & F \\
\hline 5. & mAwq & F \\
\hline 6. & xiS & F \\
\hline 7. & rAma & M \\
\hline 8. & hari & M \\
\hline 9. & SamBu & M \\
\hline 10. & Bavaw & M \\
\hline 11. & Piwq & M \\
\hline 12. & XAwq & M \\
\hline 13. & Sarva & M \\
\hline 14. & suhqw & M \\
\hline 15. & guNin & M \\
\hline 16. & rAjan & M \\
\hline 17. & rAj & M \\
\hline 18. & jFAna & N \\
\hline & & \\
\hline
\end{tabular}


International Journal on Natural Language Computing (IJNLC) Vol. 3, No.3, June 2014

\begin{tabular}{|c|l|l|}
\hline 19. & vAri & $\mathrm{N}$ \\
\hline 20. & XAwq & $\mathrm{N}$ \\
\hline 21. & wapas & $\mathrm{N}$ \\
\hline 22. & XanuR & $\mathrm{N}$ \\
\hline 23. & nAman & $\mathrm{N}$ \\
\hline
\end{tabular}

The 21 inflectional forms belonging to seven cases (excluding vocative case) of the above mentioned words were collected to serve as paradigms and stored in a systematic way as indicated below:

Table 2: Paradigm Table

\begin{tabular}{|l|l|l|l|l|l|l|}
\hline Word & Gender & Inflected Form & Remove & Add & Case & Number \\
\hline rAma & $\mathrm{M}$ & rAmayoH & ayoH & a & $6 \# 7$ & 2 \\
\hline rAma & $\mathrm{M}$ & rAmAByAm & AByAm & a & $3 \# 4 \# 5$ & 2 \\
\hline rAma & $\mathrm{M}$ & rAmasya & asya & a & 6 & 1 \\
\hline rAma & $\mathrm{M}$ & rAmO & O & a & $1 \# 2$ & 2 \\
\hline rAma & $\mathrm{M}$ & rAmam & am & a & 2 & 1 \\
\hline rAma & $\mathrm{M}$ & rAmaH & aH & a & 1 & 1 \\
\hline rAma & $\mathrm{M}$ & rAmeRu & eRu & a & 7 & 3 \\
\hline rAma & $\mathrm{M}$ & rAmeNa & eNa & a & 3 & 1 \\
\hline rAma & $\mathrm{M}$ & rAmEH & EH & a & 3 & 3 \\
\hline rAma & $\mathrm{M}$ & rAmeByaH & eByaH & a & $4 \# 5$ & 3 \\
\hline rAma & $\mathrm{M}$ & rAme & e & a & 7 & 1 \\
\hline rAma & $\mathrm{M}$ & rAmAya & Aya & a & 4 & 1 \\
\hline rAma & $\mathrm{M}$ & rAmAw & Aw & a & 5 & 1 \\
\hline rAma & $\mathrm{M}$ & rAmANAm & ANAm & a & 6 & 3 \\
\hline rAma & $\mathrm{M}$ & rAmAn & An & a & 2 & 3 \\
\hline rAma & $\mathrm{M}$ & rAmAH & AH & a & 1 & 3 \\
\hline
\end{tabular}

The first field in the above table indicates the base form or stem, the second field indicates the gender, the third field indicates the surface form or inflection, the fourth field indicates the suffix to be removed, the fifth field indicates the suffix to be added, the sixth field indicates the vibhakti, the seventh field indicates the vacanam. The contents of Paradigm table are stored in a text file. Each column is separated with a "\$" symbol. The suffixes from the Paradigm Table have been extracted from all these inflections and recorded separately. The suffix in the surface form or the input is identified from the Suffix Info table. Then a simple lookup was done on Paradigm Table for the suffix. The suffix part from the surface form will be stripped and replaced with the $5^{\text {th }}$ field in the Paradigm Table to generate the stem of the given word. Simultaneously the gender, case and number information may also be obtained from $2^{\text {nd }}, 6^{\text {th }}$ and $7^{\text {th }}$ fields from the Paradigm Table. There may be a chance of getting more than one stem for the given word. In this case all the stems that were produced were considered as candidates for the second phase. Figure 7 illustrates the idea of finding the base form from the input word which is in surface form. 


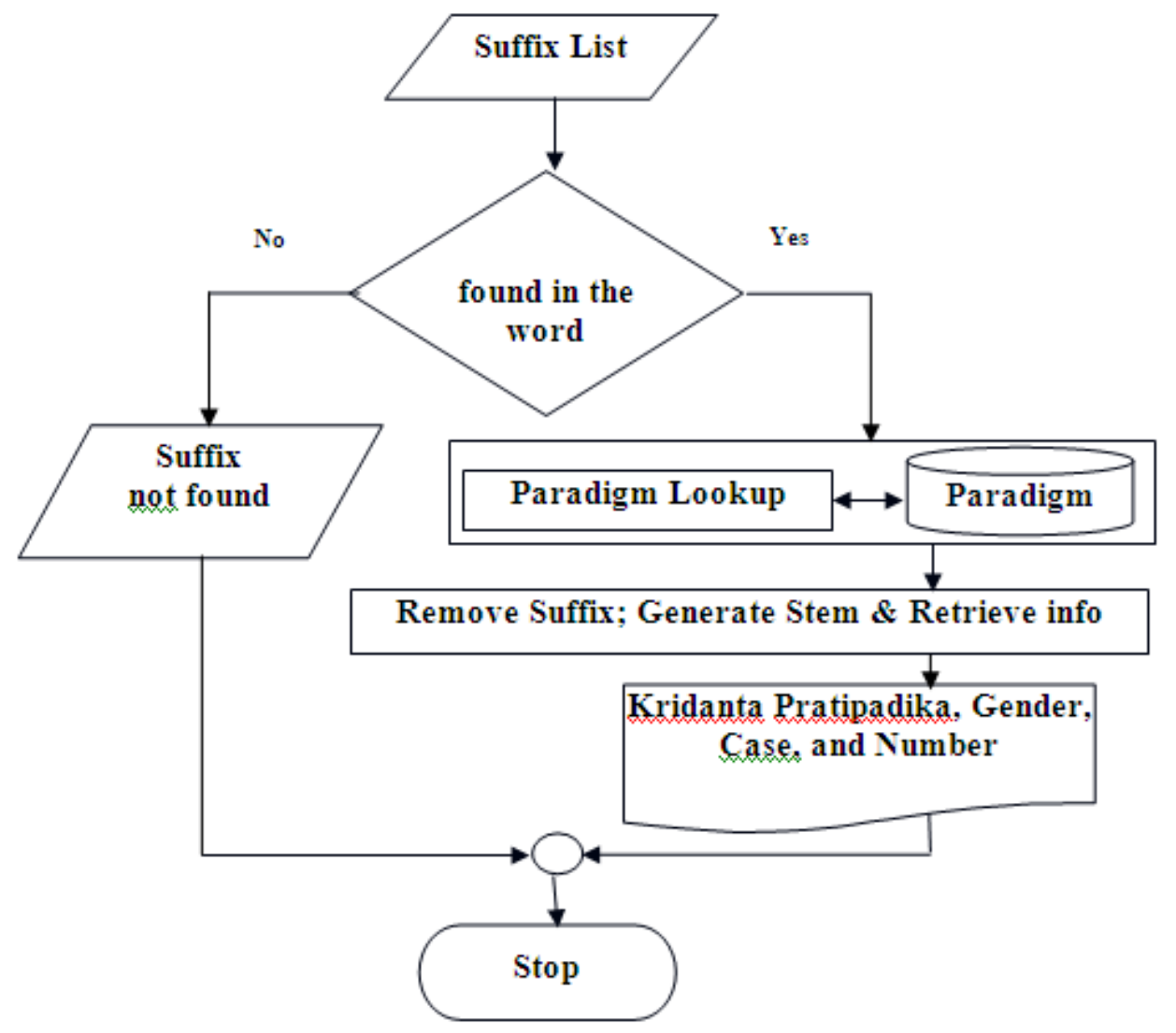

Figure 7. Stemmer and Feature Extractor

The surface form is AgawavAn (Agatavan). For this surface from the Stemmer and Feature extractor has given two possible pratipadikas, gender, case and number. The output is separated with "/" symbol. The first part indicates the unrecognized word. The second part gives various possible stems of the unrecognized word along with its gender, case and number. Each possible solution is separated with "\%" symbol. The pratipadikam, gender, case and number are separated with a comma. The output of the Stemmer and Feature Extractor for Sanskrit is given below.

Input word: AgawavAn

Output:

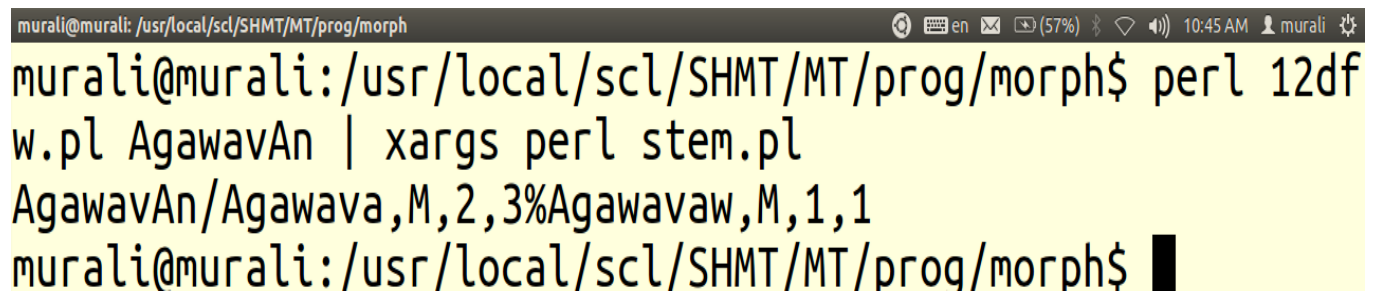

Figure 8. Output of the Stemmer and Feature Extractor

The information produced in this first phase was passed to the second phase to ensure whether the given word is kridanta or not. The base form along with the other features are passed to the Kridanta Analyzer for the analysis in order to find the encoded information regarding root, krit 
suffix, upapada and upasarga for all possible base forms listed by the Stemmer and Feature Extractor.

\subsection{Kridanta Analyzer}

Kridanta Analyzer deals fully with the kridantas except $u_{s} i d i$ suffixes and suffixes related to Vedic svaris. It is mentioned earlier that kridantas may be nouns or avyayas or adjectives. Except tumun, ktvi, amul and lyap the other kait pratyayas are used to derive nouns from verbs. These four suffixes are used to form avyayas or indeclinable forms which were discussed in the previous section. Kridanta Analyzer will give the derivational morphological information of a kaidanta i.e., the upapada, upasarga, root and suffix information. Linguistic information for upapada, upasarga, kait has been represented in the format as mentioned in Section IV - A. The following figure illustrates the functioning of Kridanta Analyzer.

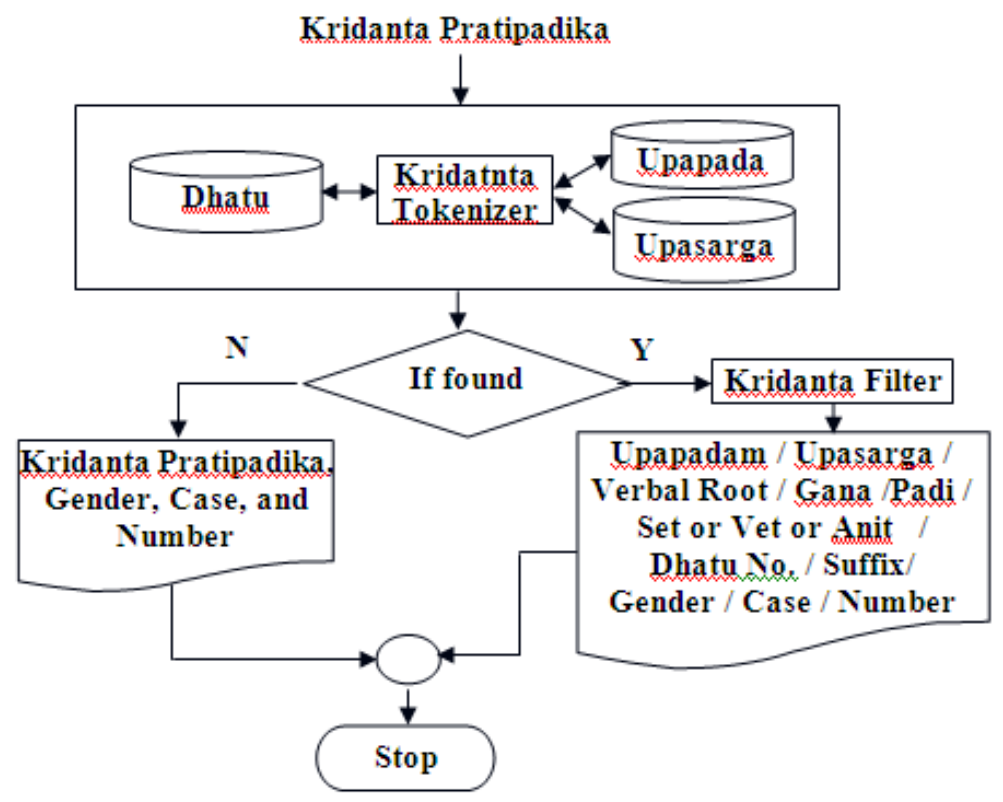

Figure 9. Kridanta Analyze

\subsubsection{Identification of kridantas}

To check whether the given word is kridanta or not, identify the pratipadika (stem) of the given word as mentioned in the previous section and this pratipadikam will be the input for the Kridanta Analyzer. First, it checks whether the given word is a kridanta and if it does not contain any upapada or upasagra, then it gives the root and suffix information. If it fails to analyze the kridanta, it means that the given word may contain any upapada or upasarga or both along with the root and suffix or the word may not be a kridanta. As upapada and upasarga are optional, they may or may not present in the given word. Hence, if upapada exists then it returns the upapada info and strips it, or else it checks whether upasarga exists or not. If upasarga is found, then returns upasarga info and then strips it. Finally it checks the root and suffix and returns the same. This process will continue for all possible candidate solutions generated in the first phase. The sample output of the Kridanta Analyzer is given below.

Input: aBijFAwam (abhijnatam)

Output: 
muraliemurali:/usr/local/scl/SHMT/MT/prog/morph\$ perl 12df w.pl aBijfAwam I xargs perl stem.pl | xargs perl 27octkqw. pl

<Word: aBijFAwam><vargaH: KQ><lifgam: M><viBakwiH: $2><$ vaca nam: 1><level: 1><upapaxam: xxx><upasargaH: aBi $><$ prAwipaxi kam: aBijFAwam/aBijFAwa $><X A w u H:$ jFA9_jFA $><$ prawyaya: kwa\#>< gaNaH: 9><paxI: pa>

<lifgam: M><viBakwiH: 2><vacanam: 1><level: 1><upapaxam: n aF><upasargaH: $x x x><p r A w i p a x i k a m: a B i j F A w a m / B i j F A w a><X A w u H$ : jFA9_jFA $><$ prawyaya: kwa\#><gaNaH: 9><paxI: pa> murali@murali:/usr/local/scl/SHMT/MT/prog/morph\$

Figure 10. Output of the Kridanta Analyzer

Input: aBijFAwavAn (abhijnatavan)

Output:

murali@murali:/usr/local/scl/SHMT/MT/prog/morph\$ perl 12df w.pl aBijFAwavAn | xargs perl stem.pl | xargs perl 27octkq W.pl

<Word: aBijFAwavAn><vargaH: KQ $><$ lifgam: M $><$ viBakwiH: $1><$ va canam: 1><level: 1><upapaxam: xxx><upasargaH: aBi $><p r A w i p a$ xikam: aBijFAwavAn/aBijFAwavaw $><$ XAwuH: jFA9_jFA $><$ prawyaya: kwavawu\#><gaNaH: 9><paxI: pa>

<lifgam: M><viBakwiH: 1><vacanam: 1><level: 1><upapaxam: n aF><upasargaH: xxx><prAwipaxikam: aBijFAwavAn/BijFAwavaw $><$ XAwuH: jFA9_jFA><prawyaya: kwavawu\#><gaNaH: 9><paxI: pa> murali@murali: /usr/local/scl/SHMT/MT/prog/morph\$

Figure 11. Output of the Kridanta Analyzer

Input: aBinayanwI (abhinayanti)

Output

muraliemurali:/usr/local/scl/SHMT/MT/prog/morph\$ perl 12df w.pl aBinayanwI | xargs perl stem.pl | xargs perl 27octkqw .pl

<Word: aBinayanwI ><vargaH: KQ><lifgam: F $><$ viBakwiH: $1><$ vac anam: 1><level: 1><upapaxam: xxx><upasargaH: aBi $><$ prAwipax ikam: aBinayanwI/aBinayanwI $><$ XAwuH: NIf1_nI $><$ prawyaya: Saw q\#Sawq $><$ gaNaH: 1><paxI: u>

<lifgam: F><viBakwiH: 1><vacanam: 1><level: 1><upapaxam: n aF><upasargaH: $x x x><$ prAwipaxikam: aBinayanwI/BinayanwI $><X A$ wuH: NIf1_nI ><prawyaya: Sawq\#Sawq><gaNaH: 1><paxI: u> murali@murali:/usr/local/scl/SHMT/MT/prog/morph\$

Figure 12. Output of the Kridanta Analyzer

Input: AcaranwI (Acaranti)

Output:

murali@murali:/usr/local/scl/SHMT/MT/prog/morph\$ perl 12df w.pl AcaranwI | xargs perl stem.pl | xargs perl 27octkqw.p l

<Word: AcaranwI ><vargaH: KQ><lifgam: F><viBakwiH: 1><vacan am: 1><level: 1><upapaxam: xxx><upasargaH: Af ><prAwipaxika

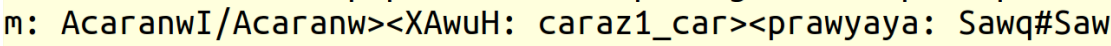
$q><$ gaNaH: $1><$ paxI : pas

<Word: AcaranwI ><vargaH: kQ><lifgam: F><viBakwiH: 1><vacan am: 1><level: 2><upapaxam: xxx><upasargaH: Af $><p r$ Awipaxika

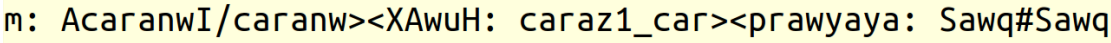
$><$ gaNaH: 1><paxI: pa>

Figure 13. Output of the Kridanta Analyzer

Failure of recognizing a kridanta may occur in two cases: 
If the upapada / upasargas not found in the table

If the given word is not a kridanta

In first case, Kridatna Analyzer has an efficient mechanism to deal with such type of words. First the word will be matched from the right hand side, letter by letter till the maximum match is found. Then the first part which is not recognized by the Kridanta Analyzer will be treated as "pUrvapaxam" (purvapadam) and the analysis for the remaining portion of the word that was recognized by the Kridatna Analyzer will be presented as specified above. Consider the following example.

Input Word: AgamanakAraNam (Agamanakaranam)

In the first iteration Kridanta Analyzer cannot directly analyze the above mentioned word. The reason is the word "Agamana" is not available in the upapda table. Hence, the Kridanta Analyzer will start analyzing the word letter by letter from the end of the word towards the beginning of the word. Now Kridanta Analyzer will take the maximum matching part as the final solution. From the given input word, the unmatched portion will be treated as purvapadam and the analysis for the maximum matched portion will be considered as the candidate solution. The analysis for the given input word "AgamanakAraNam" is given below:

Input Word: AgamanakAraNam（igamanakira ${ }_{s}$ am)

Output:

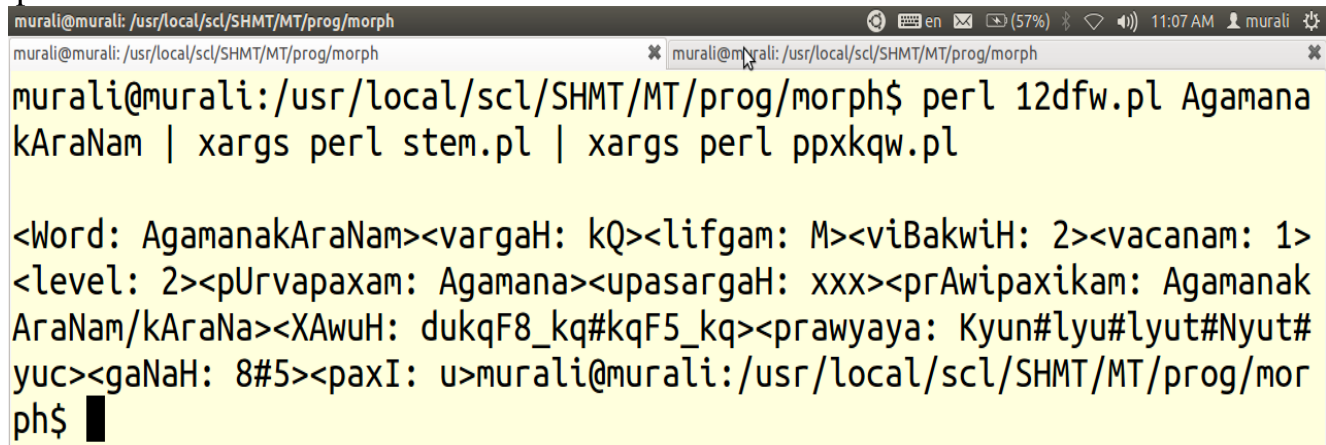

Figure 14. Output of the Kridanta Analyzer

Input Word: aKAlamaraNam (akalamaranam)

Output:

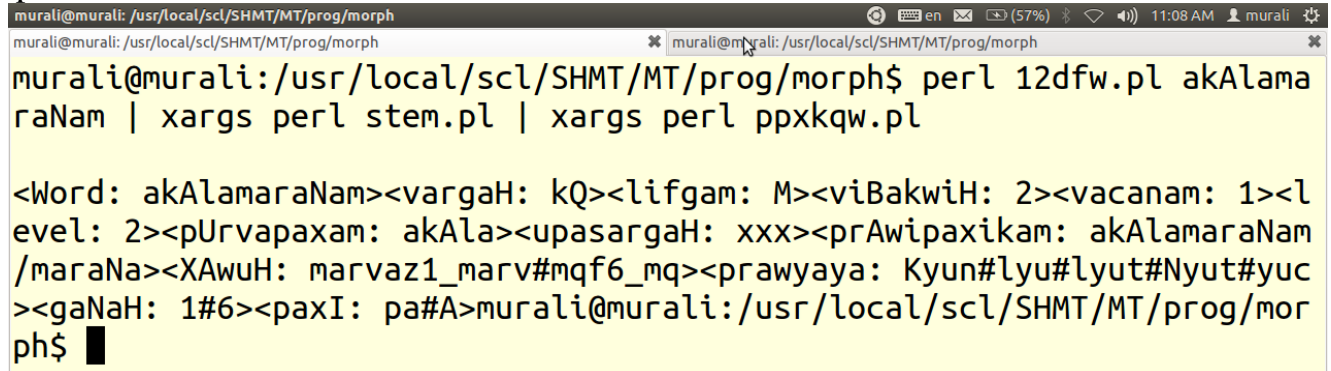

Figure 15. Output of the Kridanta Analyzer

In the second case, the basic information generated by the Stemmer and Feature Extractor regarding gender, case, number will be returned by the Kridanta Analyzer. 
International Journal on Natural Language Computing (IJNLC) Vol. 3, No.3, June 2014

\section{Performance Evaluation of Avyaya Analyzer and Kridanta ANALYZER}

Precision and Recall are the two measures that are widely used to evaluate NLP systems [7]. The Avyaya Analyzer and Kridanta Analyzers have been tested with another test data collected from Sanskrit Consortium, University of Hyderabad. Total number of words is 4573, out of these 534 words are classified as kridantas, 99 words are classified as avyayas. The precision and recall of both the tools are given in table 3 .

Table 3: Precision and Recall of the tools developed

\begin{tabular}{|c|c|c|c|c|}
\hline Tool & \begin{tabular}{|c|} 
Total No. \\
of words \\
Tested
\end{tabular} & \begin{tabular}{|c|} 
Correctly \\
Recognize \\
d Words \\
(A) \\
\end{tabular} & \begin{tabular}{|c|}
$\begin{array}{c}\text { Unrecognize } \\
\text { d Words }\end{array}$ \\
(B) \\
\end{tabular} & $\begin{array}{c}\text { Wrongly } \\
\text { Recognize } \\
\text { d Words } \\
(C) \\
\end{array}$ \\
\hline \begin{tabular}{|l|} 
Avyaya \\
Analyzer \\
\end{tabular} & 99 & 73 & $\overline{18}$ & 08 \\
\hline \begin{tabular}{|l|} 
Kridanta \\
Analyzer \\
\end{tabular} & 534 & 474 & $\overline{23}$ & 37 \\
\hline
\end{tabular}

Precision $=(\mathrm{A} /(\mathrm{A}+\mathrm{C})) \mathrm{X} 100$

Recall $=(\mathrm{A} /(\mathrm{A}+\mathrm{B})) \mathrm{X} 100$

Precision and Recall for Avyaya Analyzer is measured to 90.123\% and $80.219 \%$. Precision and Recall for Kridanta Analyzer is measured to $92.75 \%$ and $95.37 \%$.

\section{CONCLUSION}

In Sanskrit both kridantas and avyayas play an important role in a given sentence. At elementary level of Sanskrit and daily conversation one can find that kridanta and avyaya have been used very frequently. Hence without the analysis of kridanta and avyaya, it is impossible to understand a sentence properly. The advantages of Avyaya Analyzer and Kridanta Analyzers are given below.

- The Kridanta Analyzer and Avyaya Analyzer have been developed using PERL. Hence, these tools can be integrated with any MA with slight modifications for the analysis of avyayas and kridantas.

- Stemmer and Feature Extractor, Avyaya Analyzer and Kridanta Analyzer can process a single word or a file. All these three tools are integrated through a shell script. If all these three tools are improved further these tools can be used as an independent MA.

There are certain limitations with both the analyzers.

- The language filter must be improved to filter the solutions which are not required.

- In some cases the Kridanta Aanlyzer may give wrong gender information.

- Both the analyzers may fail in analyzing certain Kridantas which belongs to nic

- Complete Taddhita Analysis is yet to be done. 


\section{ACKNOWLEDGMENT}

We sincerely thank Dr. Amba P. Kulkarni, Department of Sanskrit Studies, University of Hyderabad who has given consent to study the tools developed by her. We extend our gratitude to Dr. Ankil Kumar, IIIT, Hyderabad and Mr. Narayana, R.S.V.P., Tirupati who helped us in testing the tools developed by us.

\section{REFERENCES}

[1] K.V. Abhyankar. (1961). A Dictionary of Sanskrit Grammar. Oriental Institute of Baroda.

[2] Chakradhar Nautiyaalhansa Shastri. (1966). Brihadanuvadachandrika. Motilal Banarsidas, ND.

[3] Lovins, J. B. (1968). Development of a Stemming Algorithm. Mechanical Translation and Computational Linguistics, vol.11, nos. 1 and 2.

[4] Porter, M. F. (1980). An algorithm for suffix stripping. Program: electronic library and information systems, 14(3), 130-137.

[5] Jha, Ramachandra. (Eds.). (2007). Rupachandrika. Chaukhambha Sanskrit Series Office. Varanasi.

[6] Dr. R.V.R. Krishna Sastri. (1997). Samskritavyakaranam. Krishnanada Mutt, Hyderabad

[7] Melamed, I. D., Green, R., \& Turian, J. P. (2003, May). Precision And Recall Of Machine Translation. In Proceedings Of The 2003 Conference Of The North American Chapter Of The

Association For Computational Linguistics On Human Language Technology: Companion Volume Of The Proceedings Of HLT-NAACL 2003, Short Papers, Vol. 2, pp. 61-63. Association For Computational Linguistics.

[8] Daniel Jurafsky And James H. Martin. (2004). Speech And Language Processing. Pearson Education. New Delhi.

[9] Jayan, J. P., Rajeev, R. R., \& Rajendran, S. (2009). Morphological Analyser For Malayalam-A Comparison Of Different Approaches. IJCSIT, 2(2), pp. 155-160.

[10] Menaka, S., \& Sobha, L. (2009). Optimizing The Tamil Morphological Analyzer.

[11] Parakh, M., \& Rajesha, N. (2011). Developing Morphological Analyzers For Four Indian Languages Using A Rule Based Affix Stripping Approach. In Proceedings Of Linguistic Data Consortium For Indian Languages, CIIL, Mysore.

[12] Murali N., Ramasree RJ. (April, 2011). Kridanta Analyzer. In proceedings of Annual International Conference on Emerging Research Areas. Organized by Amal Jyothi College of Engineering, Kerala. pp. 63-66

[13] Murali, N, Ramasree, R. J., \& Acharyulu, K. V. R. K. (2012). Avyaya Analyzer: Analysis of Indeclinables using Finite State Transducers. International Journal of Computer Applications, 38(6), 7-11.

[14] Murali N, Ramasree RJ. (November, 2013). Rule-based Extraction of Multi-Word Expressions for Elementary Sanskrit Texts. International Journal of Advanced Research in Computer Science and Software Engineering - Volume 3, Issue 11, pp. 661-667. ISSN: 2277 128X

[15] Murali N., Ramasree RJ. (April, 2011). Kridanta Analyzer. In proceedings of Annual International Conference on Emerging Research Areas. Organized by Amal Jyothi College of Engineering, Kerala. pp. 63-66

[16] Murali, N, Ramasree, R. J., \& Acharyulu, K. V. R. K. I (2012). Avyaya Analyzer: Analysis of Indeclinables using Finite State Transducers. International Journal of Computer Applications, 38(6), 7-11. 
International Journal on Natural Language Computing (IJNLC) Vol. 3, No.3, June 2014

Authors

N. Murali

Department of Computer Science,

Sri Venkateswara Vedic University, Tirupati - 517502

email: murali.nandi@gmail.com

Website: www.pp16-17.org

Prof. R.J. Ramasree

Department of Computer Sicence,

Rashtriya Sanskrit Vidypaeetha, Tirupati - 517507

email: rjramasree@yahoo.com

Blog: http://rjramasree.blogspot.in/p/research-publications.html

Prof. K.V. Ramakrishnamacharyulu

Professor of Vyakarana,

Department of Vyakarana (Retired),

Rashtriya Sanskrit Vidypaeetha, Tirupati - 517507

email: kvrkus@gmail.com

Website: www.pp16-17.org
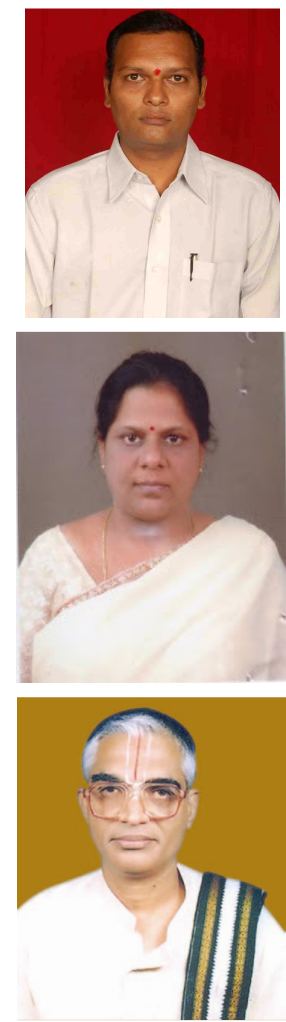